\section{(6) OPEN ACCESS}

\title{
HIV prevalence and related risk behaviours among female sex workers in Iran: results of the national biobehavioural survey, 2010
}

\author{
Leily Sajadi, ${ }^{1}$ Ali Mirzazadeh, ${ }^{1,2}$ Soodabeh Navadeh, ${ }^{1}$ Mehdi Osooli, ${ }^{1,3}$ \\ Razieh Khajehkazemi, ${ }^{4}$ Mohammad Mehdi Gouya, ${ }^{5}$ Noushin Fahimfar, ${ }^{6}$ \\ Omid Zamani, ${ }^{1}$ Ali-Akbar Haghdoost ${ }^{1,4}$
}

\begin{abstract}
translation
For numbered affiliations see end of article
\end{abstract}

\section{Correspondence to} Razieh Khajehkazemi, Research Center for modeling in Health, Institute for Futures Studies in Health, Kerman University of Medical Sciences, Ebn-e-Sina Ave, Jahad Blvd, Kerman 7619813159, Iran; r.khajehkazemi@gmail.com

Received 22 July 2013 Revised 17 September 2013 Accepted 20 September 2013

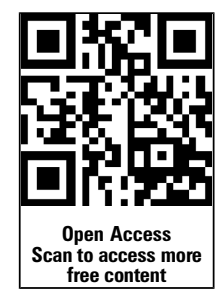

To cite: Sajadi $L$, Mirzazadeh A, Navadeh S, et al. Sex Transm Infect 2013;89:iii37-iii40.

\section{ABSTRACT \\ Objectives To determine the prevalence of HIV and related behavioural risks among Iranian female sex workers (FSW) via the first national biobehavioural surveillance survey.}

Methods In 2010, 1005 FSW were approached and 872 recruited using facility-based sampling from 21 sites in 14 cities in Iran. We collected dried blood samples and conducted face-to-face interviews using a standardised questionnaire. Data were weighted based on the response rate and adjusted for the clustering effect of the sampling site. Adjustment was performed by weighting based on the sampling fraction of each site using a prior estimate of its total size of the FSW population.

Results The prevalence of HIV infection $(95 \% \mathrm{CI})$ was $4.5 \%$ (2.4 to 8.3 ) overall, $4.8 \%$ (2.2 to 9.8) among those who had reported a history of drug use and $11.2 \%$ (5.4 to 21.5) among those who had a history of injection drug use. The frequencies of condom use in the last sexual act with paying clients and non-paying partners were $57.1 \%$ and $36.3 \%$, respectively. Any drug use was reported by $73.8 \%$ of participants, and among this subgroup, $20.5 \%$ had a history of injection drug use.

Conclusions The prevalence of HIV was considerable among FSW particularly those who had a history of drug injection. A combination of prevention efforts addressing unsafe sex and injection are needed to prevent further transmission of HIV infection.

\section{INTRODUCTION}

Iran's HIV epidemic has been characterised by the spread of infection among people who inject drugs (PWID) with dramatic outbreaks noted in prisons in the mid 1990 s. $^{1}$ Such outbreaks pushed health authorities to scale up the HIV response by expanding harm reduction services in and outside prisons, voluntary counselling and testing (VCT) sites for HIV (from 231 sites in 2006 to 476 in 2010), and sexually transmitted disease (STD) clinics serving vulnerable women including female sex workers (FSW). These efforts led to an increase in the number of identified HIV cases to 23497 cases by 2012, with the dominant mode of transmission still drug injection (69.8\%). The case notifications, however, indicated an increasing contribution of unsafe sex-up to $20.8 \%$ for those cases identified in $2010 .^{2}$
Based on expert opinion, it is estimated that overall, 80000 active FSW are living in Iran, ${ }^{3}{ }^{4}$ while $7 \%$ of men aged between 18 to 45 have had sexual contact with a FSW over the last year. ${ }^{5} \mathrm{~A}$ recent mathematical modelling exercise estimated that approximately $1100 \mathrm{FSW}$ in Iran could be infected with HIV by $2014 .^{6}$ However, the epidemiology of HIV and high risk behaviours among FSW are not yet well studied due to the highly stigmatised and illegal nature of sex work, and further of any sex outside of marriage in Iran. The aim of this first national biobehavioural surveillance survey among FSW was to assess the prevalence of HIV infection and related risky behaviours in this special subpopulation.

\section{METHODS}

This study was envisioned as the first round of ongoing biobehavioural surveillance surveys ${ }^{7}$ for the FSW populations in Iran, and was conducted between April and July 2010 in 21 sites in 14 cities. The cities were chosen to be geographically representative of all regions in the country. The recruiting sites were a combination of nongovernmental organisations and public STD clinics serving vulnerable women (13 sites) and drop-in centres serving men and women, including FSW (8 sites). Given the local health department surveillance team inputs, we chose a maximum of two facilities per city for the study, according to the capacity of staff to consent, enrol and interview the participants.

Of those seeking routine services at each recruiting site, 30-45 eligible FSW were recruited using convenience sampling. FSW were approached by a trained (peer) recruiter who verified eligibility criteria (age $\geq 18$ years, selling sex for money, drugs, or goods in the last 12 months, and a history of practising sex work for at least 6 months) and explained the study benefits and potential harms. The study protocol and procedures were reviewed and approved by the Research Review Board at Kerman University of Medical Sciences (2010-no. 90/122).

Our questionnaire on demographic characteristics, history of sex work, and related sex and drug use risks was based on previous questionnaires developed by Family Health International ${ }^{3}$ with inclusion of other local and international indicators. Among additional indicators included were engagement in temporary marriage (Sighe), local 
venues that FSW use for either finding clients or having sex, history of being in prison and knowledge of available sites for HIV testing. We also asked about sexually transmitted infection syndromes and treatment seeking patterns. After obtaining verbal informed consent, every FSW was anonymously interviewed by a trained interviewer. Upon providing a separate consent for HIV testing, a dried blood spot (DBS) specimen was also collected. An incentive (US \$2; except in Tehran, which was US \$3) was given as a gift for participation. A unique 10-digit code linked each questionnaire and its corresponding DBS. Every participant was able to obtain her HIV test result after providing the unique identifier code to the consulter either at the recruitment site or in the neighbourhood local VCT site. Positive cases were linked to services based on routine ongoing procedures at VCT centres.

HIV seropositivity was assessed using ELISA. Each DBS was considered positive if it had two ELISA reported results that were higher than the cut-off point. The first ELISA was performed using a bio Mérieux Vironostika Uni-Form II Ag/Ab kit; for the second test, a Bio-Rad Genscreen Plus HIV Ag-Ab kit was used. A third test was performed for discordant results. Quality control testing was performed by the reference laboratory (Pasteur Institute of Iran) on 10\% of samples randomly selected from all specimens.

Data were weighted and adjusted for the clustering effect of the sampling sites. Since we recruited FSW from the sites, we considered the sites as sampling units to take into account such clustering effects in our analysis. Each site was serving a different number of FSW, but we recruited a fixed number of 30-45 FSW per site. To adjust for the differential sampling probabilities, we conducted a weighted analysis. Point estimates and 95\% CIs were calculated. The $\chi^{2}$ test was used to assess correlates of HIV infection. Statistical significance was set at $\mathrm{p}<0.05$. All analyses were performed using STATA V.10 (Stata Corp.).

\section{RESULTS}

We initially approached $1005 \mathrm{FSW}$; from that group the entire dataset from one province (100 FSW) was excluded mainly because of low-quality data collection based on the feedback of the provincial supervisor and the results of our external evaluation. From the remaining 12 provinces, $32 \mathrm{FSW}$ were excluded because of ineligibility and 1 because of refusal to give consent. Of 872 eligible FSW that consented for the interview, 817 (93.7\%) also consented for blood draw.

The mean age of the respondents was 31.8 years (SD 9.1, median 30 years). Ever being married was reported by $83.2 \%$, while $35.8 \%$ were married at the time of the interview. About half $(46.5 \%)$ had no education beyond primary school. The mean age at first commercial sex was 24.6 years (SD 7.6, median 24 years). Less than half of FSW (41.6\%) had engaged in sex work for more than 4 years. Having another source of income other than sex work was reported by $36.5 \%$.

The average number of paying clients reported by FSW in the last 7 days was 3.1 (SD 3.9, median 2 clients). Approximately one-fifth $(22.1 \%)$ of FSW had never used condoms in any type of sexual contact. Overall, $57.1 \%$ of FSW had used condoms in their last sexual contact with a paying client, while $49.1 \%$ reported consistent condom use with paying clients over the last month prior to the interview. Of those who reported having a non-paying partner $(57.2 \%$ of the total), $36.3 \%$ had used a condom during the last sexual act and $28 \%$ reported consistent condom use with the non-paying partners over the last month prior to the survey.
A total of $73.8 \%$ reported a history of any drug use. Of these, 63.2\% were active drug (other than methadone) users at the time of the survey. Among those with a history of drug use, intravenous drug injection was reported by $20.5 \%$, and $26.6 \%$ of these were active injecting drug users at the time of the survey. The mean ages for first drug use and first drug injection were 21.5 years (SD 7.1, median 20 years) and 25.8 years (SD 8.3 , median 25 years), respectively. The percentage of FSW who reported high-risk injection (ie, shared syringes or equipment) at their last injection was $11.6 \%$.

We found 30 HIV-positive samples. The overall prevalence of HIV infection was $4.5 \%$ (95\% CI 2.4 to 8.3 ). HIV prevalence varied substantially between the different sites/cities with a range of $0 \%$ to $28.6 \%$, but with wide and overlapping CIs. The prevalence of HIV was $4.8 \%$ among FSW who reported a history of any drug use, and $11.2 \%$ for those who had a history of drug injection. We found that the prevalence of HIV infection was significantly associated with having a history of drug injection and having another source of income in addition to sex work. HIV prevalence by several indicators of risk behaviour is presented in table 1 .

Table 1 Demographic and behavioural characteristics and the weighted HIV prevalence among FSW in Iran, 2010

\begin{tabular}{|c|c|c|c|}
\hline Behavioural factors & $\mathrm{N}(\%)$ & HIV prevalence $(95 \% \mathrm{CI})^{*}$ & p Valuet \\
\hline \multicolumn{4}{|l|}{ Current age } \\
\hline $18-24$ & $210(23.8)$ & 0 & \multirow[t]{3}{*}{0.085} \\
\hline $25-34$ & $362(41.7)$ & 5.8 (2.9 to 11.4$)$ & \\
\hline$\geq 35$ & $292(34.5)$ & 6.0 (2.8 to 12.3$)$ & \\
\hline \multicolumn{4}{|l|}{ Current marital status } \\
\hline Married/ ever married & $697(81.6)$ & 5.0 (2.6 to 9.5$)$ & \multirow[t]{2}{*}{0.224} \\
\hline Single & $172(18.4)$ & $1.9(0.4$ to 8.9$)$ & \\
\hline \multicolumn{4}{|l|}{ Age at first commercial sex } \\
\hline$\leq 15$ & $47(7.7)$ & 0 & \multirow[t]{4}{*}{0.132} \\
\hline $16-20$ & $223(28.8)$ & 3.7 (1.1 to 11.6$)$ & \\
\hline $21-25$ & $216(25.9)$ & $1.6(0.4$ to 5.6$)$ & \\
\hline$\geq 26$ & $313(37.6)$ & 7.6 (3.4 to 16.1$)$ & \\
\hline \multicolumn{4}{|c|}{ Having another source of income in addition to sex work } \\
\hline Yes & $298(36.5)$ & 7.1 (3.5 to 13.9) & \multirow[t]{2}{*}{0.027} \\
\hline No & $550(63.5)$ & 3.2 (1.5 to 6.8$)$ & \\
\hline \multicolumn{4}{|c|}{ Number of paying clients in the last 7 days } \\
\hline None & $440(53.9)$ & $5.2(2.7$ to 9.8$)$ & \multirow[t]{3}{*}{0.185} \\
\hline $1-2$ & $233(27.9)$ & $2.6(0.9$ to 7.2$)$ & \\
\hline$\geq 2$ & $151(18.2)$ & $2.1(0.4$ to 9.6$)$ & \\
\hline \multicolumn{4}{|c|}{ Condom use during last sexual act with paying clients } \\
\hline Yes & $531(57.1)$ & $3.9(1.9$ to 7.8$)$ & \multirow[t]{2}{*}{0.405} \\
\hline No & $310(42.9)$ & $5.6(2.4$ to 12.8$)$ & \\
\hline \multicolumn{4}{|l|}{ Having non-paying partners } \\
\hline Yes & $481(57.2)$ & 4.7 (2.2 to 9.8$)$ & \multirow[t]{2}{*}{0.734} \\
\hline No & $389(42.8)$ & 4.1 (1.9 to 8.7$)$ & \\
\hline \multicolumn{4}{|l|}{ Having ever used drugs } \\
\hline Yes & $624(73.8)$ & 4.8 (2.2 to 9.8$)$ & \multirow[t]{2}{*}{0.307} \\
\hline No & $247(26.2)$ & 2.8 (1.3 to 5.9$)$ & \\
\hline \multicolumn{4}{|c|}{ Having ever injected drugs ${ }^{\ddagger}$} \\
\hline Yes & $127(20.5)$ & 11.2 (5.4 to 21.5$)$ & \multirow[t]{2}{*}{0.003} \\
\hline No & $497(79.5)$ & 3.1 (1.5 to 6.4$)$ & \\
\hline
\end{tabular}




\section{DISCUSSION}

The prevalence of HIV in our first national biobehavioural surveillance survey of FSW was $4.5 \%$, considerably higher than the no infections $(0.0 \%)$ and $2.7 \%$ prevalence measured in previous surveys among FSW in Iran. ${ }^{8}$ In a recent survey among FSW in Shiraz using respondent driven sampling, HIV prevalence was reported at $4.7 \%{ }^{9}$ which is comparable to what we observed in our study. It is of concern that we have documented high levels of drug use and injection drug use in this population. The high rate of drug injection in this sample illustrates a link between FSW and PWID; the population most severely affected by HIV in Iran. HIV prevalence among FSW who injected drugs was more than three times that among those who did not inject drugs, and it was comparable to HIV prevalence among PWID in Iran (15.1\%). ${ }^{2}$ The high prevalence of injection drug use in our study was also similar to another study among FSW in Iran, ${ }^{10}$ corroborating the role of drug injection in the spread of HIV among and between other high-risk groups in Iran. While it is reasonable to assume that a substantial fraction of HIV infections among FSW were acquired through drug injection rather than unprotected sex, the potential for further onward transmission from FSW to their clients and non-client partners appears high, given the rather low level of condom use among our study population. Fortunately, for now, the sexual mode of transmission in commercial sex networks does not yet appear to be a major driver of the epidemic in this country according to HIV case reporting and other estimates.

We found a lower mean number of paying clients per FSW per week in comparison with another study in Iran (5 to 9 clients), ${ }^{8}$ but this was higher in comparison to another recent study $\left(2.6\right.$ clients). ${ }^{10}$ The observed condom use during last sexual intercourse was less than that in a recent study in 2010, using respondent driven sampling to recruit 177 FSW in Kerman, which reported $83.1 \%$ condom use during last sexual contact with a client. ${ }^{10}$ In other studies in the Middle East and North Africa region, condom use in last sexual contact with paying clients varied between 24\% (FSW in Hargeisa, Somalia ${ }^{11}$ ) to $92.0 \%$ (street-based FSW in Lebanon ${ }^{12}$ ). In terms of number of clients over the last 7 days, in Somalia it was reported as a median of three paying clients, which is just above what we observed. ${ }^{11}$

FSW who had another source of income beyond selling sex were more likely to be HIV positive. This finding should be explored in further studies to examine whether such FSW are involved in other forms of risky behaviours. Incidentally, these FSW reported more non-paying partners than clients (given the fact that condom use rate is less among non-paying partners than clients; $36.3 \%$ vs. $57.1 \%$ ).

We recognise several limitations in our study. First, because the sample was recruited from health and social service facilities, the measured HIV prevalence and risk behaviours may not be representative of all FSW in Iran. Facilities are likely to include those FSW with potential health problems stemming from longer-term injection drug use or HIV infection itself. Therefore, HIV prevalence could be overestimated in our sample compared to a previous study. ${ }^{8}$ Moreover, our study was conducted over 21 sites including 13 that served FSW and other vulnerable women. The remaining eight sites were chosen purposefully to be geographically representative for all regions in the country, but feasibility was a factor in choosing specific sites within each region. To be comprehensive, we recruited from non-governmental and public health centres to capture all subgroups of the target population that are linked to services. These choices and factors may have affected the representativeness of our sample.
A recent validation study in Iran suggested that FSW tend to underestimate the number of clients and overestimate condom use with clients in the context of face to face interviews. ${ }^{13} \mathrm{~A}$ gold-standard survey methodology or truly representative sample of all FSW in the major cities of Iran is lacking, and is likely to remain a challenge for many years to come. We present the findings of the first survey of FSW in the nation as a baseline to improve upon in the future. To the extent that future surveys in the same cities, sites and facilities may reproduce comparable samples, the data presented here may serve as a basis for tracking the epidemic in this vulnerable population over time. In the meantime, our data demonstrate that HIV infection among FSW is a reality and is contributing to the overall epidemic in Iran. Multifaceted prevention interventions (ie, addressing 100\% condom use and clean syringe use) are urgently needed in Iran.

\section{Key messages}

- HIV prevalence was $4.5 \%$ among female sex workers recruited through a national study from drop-in centres, harm reduction centres and shelters.

- Female sex workers who injected drugs were more than three times as likely to be infected as those who did not inject drugs; this suggests that a considerable fraction of HIV infections among these female sex workers were acquired through drug injection.

\section{Author affiliations}

${ }^{1}$ Regional Knowledge Hub, and WHO Collaborating Centre for HIV Surveillance, Institute for Futures Studies in Health, Kerman University of Medical Sciences, Kerman, Iran

${ }^{2}$ Institute for Health Policy Studies, University of California, San Francisco, California, USA

${ }^{3}$ Centre for Thrombosis and Haemostasis, Skane University Hospital, Lund University, Malmo, Sweden

${ }^{4}$ Research Center for Modeling in Health, Institute for Futures Studies in Health, Kerman University of Medical Sciences, Kerman, Iran

${ }^{5}$ Center for Disease Control (CDC), Ministry of Health and Medical Education, Tehran, Iran

${ }^{6}$ HIV/AIDS Control Office, Ministry of Health and Medical Education, Tehran, Iran

Handling editor Jackie A Cassell

Acknowledgements The authors would like to thank the HIVIAIDS Control Office of MOH (Dr Abbas Sedaghat and Dr Kianoosh Kamali), Dr Afshin Safaie and Alborz laboratory staff, Pasteur Institute of Iran (Dr Mohammad Reza Aghasadeghi, Dr Keyhan Azadmanesh and Mr Farhang Vahabpour), the staff of Kerman Knowledge Hub for HIVIAIDS (Dr Ehsan Mostafavi, Ms Soodeh Arabnejad and Dr Farzaneh Zolala), and all supervisors and interviewers. We would also like to express our appreciation to all the women who participated in this study.

Contributors Design of the project and writing of the proposal: $\mathrm{LS}, \mathrm{A}-\mathrm{AH}, \mathrm{SN}$ $A M, M G$ and NF. Protocol writing: LS, MO, A-AH, SN, AM and OZ. Supervision of the project: A-AH. Project management and drafting the first draft of the paper: LS. Statistical analysis: RK. Interpretation of results and contributing to the development of the paper: RK, AM, SN, A-AH and MO. Review of the protocol and final manuscript: $\mathrm{MG}, \mathrm{NF}$ and $\mathrm{OZ}$.

Funding The study was supported by a grant from the United Nations Development Program (UNDP) the primary recipient of funds from the Global Funds for AIDS, Tuberculosis, and Malaria in Iran (grant number IRN-202-G01-H-00).

Competing interests None.

Ethics approval The study protocol was approved by the Ethics Committee of Kerman University of Medical Sciences.

Provenance and peer review Commissioned; externally peer reviewed.

Open Access This is an Open Access article distributed in accordance with the Creative Commons Attribution Non Commercial (CC BY-NC 3.0) license, which permits others to distribute, remix, adapt, build upon this work non-commercially, 
and license their derivative works on different terms, provided the original work is properly cited and the use is non-commercial. See: http://creativecommons.org/ licenses/by-nc/3.0/

\section{REFERENCES}

1 National HIVIAIDS committee. Third national strategic plan on HIVIAIDS draft (2009-2013). Islamic Republic of Iran, 2009.

2 National AIDS Committee Secretariatn Ministry of Health and Medical Education, Islamic Republic of Iran. AIDS Progress Report on Monitoring of the United Nations General Assembly Special Session on HIV and AIDS, March 2012. http://www. unaids.org/en/dataanalysis/knowyourresponse/countryprogressreports/2012countries/ IRIran\%20AIDS\%20Progress\%20Report\%202012\%20English\%20final1_1.pdf

3 AIDS office Ministry of Health. Modelling of new HIV infections based on exposure groups in Iran. Tehran, 2010.

4 Nasirian M, Doroudi F, Gooya MM, et al. Modeling of human immunodeficiency virus modes of transmission in Iran. J Res Health Sci 2012;12:81-7.

5 Shokoohi M, Baneshi MR, Haghdoost AA. Size estimation of groups at high risk of HIVIAIDS using Network Scale Up in Kerman, Iran. Int J Prev Med 2012;3:471-6.

6 Haghdoost AA, Mostafavi E, Mirzazadeh A, et al. Modelling of HIV in Iran up to 2014. J AIDS HIV Res 2011;3:231-9.
7 Family Health International. Behavioral Surveillance Surveys: Guidelines for repeated behavioral surveys in population at risk of HIV. http://www.who.int/hiv/strategic/en/ bss_fhi2000.pdf

8 Abu-raddad L, Akala FA, Semini I, et al., Characterizing the HIVIAIDS epidemic in the Middle East and North Africa: Time for strategic action (Orientations in Development). 1st edn. World Bank Publications, 2010.

9 Kazerooni PA, Motazedian N, Motamedifar M, et al. The prevalence of human immunodeficiency virus and sexually transmitted infections among female sex workers in Shiraz, South of Iran: By respondent-driven sampling. Int I STD AIDS Published Online First: 19 Jul 2013. doi:10.1177/0956462413496227.

10 Navadeh S, Mirzazadeh A, Mousavi L, et al. HIV, HSV2 and Syphilis prevalence in female sex workers in Kerman, South-East Iran; Using respondent-driven sampling Iranian J Pub Health 2012;41:60-5.

11 Kriitmaa K, Testa A, Osman M, et al. HIV prevalence and characteristics of sex work among female sex workers in Hargeisa, Somaliland, Somalia. AIDS 2010;24(Suppl 2):S61-7.

12 Mahfoud Z, Afifi R, Ramia S, et al. HIVIAIDS among female sex workers, injecting drug users and men who have sex with men in Lebanon: Results of the first biobehavioral surveys. AIDS 2010;24(Suppl 2):S45-54.

13 Mirzazadeh A, Haghdoost AA, Nedjat $S$, et al. Accuracy of HIV-related risk behaviors reported by female sex workers, Iran: A method to quantify measurement bias in marginalized populations. AIDS Behav 2013;17:623-31. 